\title{
APORTACIONES PARA LA EVALUACIÓN DEL PROGRAMA ECOPLATA (URUGUAY) DE GESTIÓN INTEGRADA DE ÁREAS LITORALES
}

\author{
Juan Adolfo Chica Ruiz \\ María Luisa Pérez-Cayeiro \\ Universidad de Cádiz \\ adolfo.chica@uca.es
}

\section{RESUMEN}

Si bien en las dos últimas décadas son numerosas las experiencias, a diferente escala, que se han puesto en marcha sobre Gestión Integrada de Áreas Litorales (GIAL) en el mundo, son muy pocas las que han contado con una evaluación para comprobar el grado de cumplimiento de las mismas. Es por ello que este trabajo pretende contribuir a la difusión de una de las experiencias de gestión integrada de áreas litorales más interesantes que en Iberoamérica se han dado, conocido como Programa Ecoplata (Uruguay). Por otro lado, también tiene como objetivo realizar una evaluación de la planificación y de la ejecución del citado programa, es decir, de un proceso de GIAL a largo plazo que no está exento de retos y oportunidades.

Palabras clave: áreas litorales, gestión costera, evaluación, Uruguay.

\section{ABSTRACT}

Although in the last two decades numerous programmes on different scales have been launched on Integrated Coastal Zone Management (ICZM) around the world, very few have had an evaluation to assess the degree of progress in the management model. This study has two fundamental objectives: the first of which is to contribute to the spreading of the iniciatives of integrated management's most interesting coastal areas in Iberoamérica,

Fecha de recepción: mayo 2013.

Fecha de aceptación: julio 2014. 
known as the Ecoplata Program (Uruguay). The second objective is to do an evaluation of the planning and implementation of that program, this process of long-term ICZM is not without its challenges and opportunities.

Keywords: coastal management, evaluation, Uruguay.

\section{INTRODUCCIÓN Y ANTECEDENTES}

La gestión integrada de las áreas litorales (GIAL): $1^{\circ}$ ) es un proceso de administración, $2^{\circ}$ ) que se dirige al desarrollo humano, pero al mismo tiempo, $3^{\circ}$ ) pretende conservar los recursos naturales y culturales (Barragán, 2003). Tanto en ésta como en otras definiciones (Clark, 1992; Carvalho y Rizzo, 1994; UNEP, 1995; OCDE, 1995; Cicin-Sain y Knecht, 1998; Kay y Alder, 1999; Comisión Europea, 2000; Council of Europe, 2000, a, b; UNESCO/COI, 2006) se asume, de forma implícita o explícita, que la GIAL es una herramienta al servicio de una determinada política pública. Por ésta se entiende aquello que una autoridad gubernamental decide hacer o no, ya que esto último supone también una forma de actuar. De lo anterior se deducen algunas hipótesis (Barragán, Chica, Pérez-Cayeiro, 2008):

- La gestión del litoral se inscribe dentro del marco de las políticas públicas que operan al servicio del desarrollo sostenible de estos ámbitos geográficos.

- La GIAL, como interpretación específica de un determinado modelo de gestión que se identifica en gran medida con el concepto de gobernanza.

Si se entiende la gobernanza como una forma de gobierno que tiene el objetivo de un desarrollo económico, social e institucional duradero, que promueve un sano equilibrio entre el estado, la sociedad civil y el mercado de la economía, la toma de decisiones no se basa, de forma exclusiva, en el principio de jerarquía sino además en los de participación, negociación, consenso, cooperación, etc. (Aguilar, 2006).

Por otra parte, el proceso que caracteriza la formulación de una política pública, según Olmeda (1999), implica la consecución de cinco fases: 1) identificación del problema y establecimiento en la agenda política; 2) formulación; 3) adopción de la misma; 4) implantación y 5) evaluación. Por su parte, GESAMP (1996) y Olsen, Lowry y Tobey (1999) señalan que el desarrollo de los programas gubernamentales de GIAL siguen un ciclo similar al que corresponde con el desarrollo de las grandes políticas de Estado. De acuerdo con los argumentos de estos autores, se afirma que el método de la GIAL y el ciclo descrito están plenamente relacionados.

Algunas de las ideas subyacentes que apoyan estas hipótesis son: La GIAL está basada en el ecosistema, integrando los aspectos ecológicos, sociales y económicos en todos los sectores y organismos, y entre las diferentes escalas de gobierno; la GIAL es una gestión adaptativa capaz de aprender de la experiencia; la GIAL tiene una perspectiva estratégica y con capacidad de anticipación, enfocada a largo plazo. Y por último, la GIAL requiere de la participación activa de los agentes sociales e institucionales con intereses en las áreas litora- 
les y sus recursos. En este sentido, el interés de los objetivos de este artículo se centra en dos elementos esenciales de la gestión del litoral:

A) Evaluar el Programa Ecoplata como un programa de gestión a largo plazo. Según Gee et al. (2006) con el seguimiento y la evaluación de un programa se asegura que la GIAL sea adaptativa. Por tanto, la visión estratégica en el próximo ciclo de GIAL se formulará en función del cumplimiento de los objetivos y resultados previstos.

B) Analizar de forma crítica una experiencia de gestión integrada del espacio litoral en Iberoamérica. Su finalidad es realizar una aportación al conocimiento de la gestión de las áreas litorales. Además, pretende transmitir los resultados de los procesos de gestión a los implicados e interesados. Incluso, esta acción puede constituir una referencia o servir de inspiración a los gestores y responsables de administrar recursos o ámbitos similares.

Por todo ello es conveniente reiterar que cualquier iniciativa que pueda contribuir al uso más racional de los recursos costeros debe ser bien recibida y apoyada. Como señala Barragán (2005 a) en América del Sur, Uruguay, a través de la gestión ambiental, se interesa cada vez más por la gestión específica de las zonas costeras, destacando expresamente el Programa Ecoplata.

El mejor antecedente que puede citarse se corresponde con la Evaluación del Programa de Manejo de Recursos Costeros de Ecuador, Etapa II, (PMRC II). Bajo la responsabilidad del Banco Interamericano de Desarrollo (BID) se inicia en 2012 un detallado proceso de evaluación que permite conocer detalles de método y de contenido de sumo interés (www. pmrc.gob.ec).

\section{ASPECTOS FORMALES: CONCEPTOS, MÉTODO Y FUENTES DE INFORMACIÓN}

Es preciso señalar que en la evaluación de cualquier programa de GIAL, interpretado éste como un ejercicio de política pública, conviene distinguir entre las diferentes etapas a las que va dirigida. En el caso presente se han tenido en cuenta las que otros autores (Merino, 2010; Subirats et al., 2012) proponen con un carácter genérico: Planificación (ex ante), Ejecución (in itinerem) y Final (Resultados, Impactos y Efectos). Por otro lado, y ya de una forma más específica, el método utilizado en este trabajo también se ha inspirado en evaluaciones de programas de GIAL iberoamericanos (Olsen, Lowry y Tobey, 1999; Barragán, 2003; Olsen, 2003; Olsen y Ochoa, 2004). En último lugar, otras evaluaciones realizadas por organismos internacionales (Comisión Europea, 1999; UNESCO/COI, 2001, 2003 y 2006; PNUD, 2009) también han servido de referencia. En la Tabla 1 se relacionan los aspectos del Programa Ecoplata evaluados en esta investigación (primera columna) y su pertenencia a alguna de las etapas antes mencionadas (primera fila).

Como se observa en la Tabla 1 buena parte de los aspectos evaluados se corresponden con las etapas de planificación (ex ante) y ejecución (in itinerem). Es lógico que ello sea así teniendo en cuenta que Ecoplata tiene su origen en la cooperación internacional y que, por lo tanto, no debe extrañar un ritmo más lento de avance en comparación a un proceso cuya iniciativa hubiera partido de las instituciones nacionales uruguayas. Otras aclaraciones concep- 
tuales que ayudan a comprender mejor la Tabla 1 son las que distinguen entre evaluaciones ex post hechas para a) los Resultados (outputs), que se identificarían a partir del contraste obtenido entre el Acuerdo de actuación institucional definido (Programa Ecoplata en este caso) y los actos de implementación y productos administrativos obtenidos en el proceso de desarrollo del propio programa; b) los Impactos que podrían atribuirse al Programa y que están relacionados con los cambios de conducta de los grupos objetivo (aquí se incluyen también las instituciones públicas porque la GIAL tiene en ellas especial interés debido a la necesidad de cambio en los modelos de gestión pública); y c) los Efectos reales (outcomes) del Acuerdo de actuación institucional sobre los beneficiarios finales; que en el caso de Ecoplata se puede reflejar en cambios ambientales que juegan a favor de los grupos sociales, usuarios o interesados en la costa uruguaya y sus servicios ecosistémicos.

Tabla 1

RELACIÓN ENTRE LOS ASPECTOS CONSIDERADOS EN LA EVALUACIÓN DE ECOPLATA Y LAS ETAPAS DEL PROGRAMA

\begin{tabular}{|l|c|c|c|c|c|}
\hline \multicolumn{1}{|c|}{ Aspectos evaluados } & $\begin{array}{c}\text { Planificación } \\
(\text { ex ante })\end{array}$ & $\begin{array}{c}\text { Ejecución } \\
\text { (in itinerem })\end{array}$ & $\begin{array}{c}\text { Resultados } \\
\text { (ex post) }\end{array}$ & $\begin{array}{c}\text { Impactos } \\
\text { (ex post) }\end{array}$ & $\begin{array}{c}\text { Efectos } \\
\text { (ex post) }\end{array}$ \\
\hline Equipo técnico & $\mathrm{X}$ & $\mathrm{X}$ & & & \\
\hline Integrantes y participantes & $\mathrm{X}$ & $\mathrm{X}$ & & & \\
\hline Sinergias & $\mathrm{X}$ & $\mathrm{X}$ & & & \\
\hline Planteamiento planificación & $\mathrm{X}$ & & & & \\
\hline Implicación gubernamental & & $\mathrm{X}$ & $\mathrm{X}$ & & \\
\hline Ejecución & & $\mathrm{X}$ & $\mathrm{X}$ & & \\
\hline Grupos objetivo y/o beneficiarios & & & & $\mathrm{X}$ & $\mathrm{X}$ \\
\hline Cambios institucionales & & & $\mathrm{X}$ & $\mathrm{X}$ & \\
\hline Uso de recursos & & & & $\mathrm{X}$ & \\
\hline Cambios ambientales & & & & & $\mathrm{X}$ \\
\hline
\end{tabular}

La evaluación de los aspectos seleccionados se expresa en términos cualitativos: favorable (F), medio (M) y desfavorable (D). A modo de conclusiones generales, se expone una síntesis de dicha evaluación utilizando un análisis DAFO (debilidades, amenazas, fortalezas y oportunidades).

La información básica a la que se ha tenido acceso para la realización del análisis procede de fuentes de muy distinta naturaleza:

- Bibliográfica, identificada con una serie de estudios e informes que al respecto ha editado el propio Programa Ecoplata, publicaciones de autores que trabajan en la disciplina y distintos organismos internacionales.

- Trabajo de campo en diversos municipios costeros y entrevistas con técnicos y políticos responsables de la administración de recursos costeros.

- Participación en reuniones y actividades que el Programa Ecoplata desarrolla (Mesa Ejecutiva, Junta Local del Parque del Plata, Intendencia de Maldonado, Taller de indicadores, Curso de Gestión Integrada de Zonas Costeras). 


\section{CONTEXTO. EL LITORAL DE URUGUAY Y LA GESTIÓN INTEGRADA DE LAS ZONAS COSTERAS}

Siguiendo a Barragán (2005 a y b) se puede afirmar, de una forma general, que las zonas costeras tienen una función muy relevante en Latinoamérica. En Uruguay son varias las razones que justifican dicha afirmación: a) Desde el punto de vista ambiental sustentan ecosistemas ricos y productivos que mantienen en gran medida su desarrollo económico (Viana, 2009: 9); b) concentran el 69\% de su población así como las principales actividades económicas, llegando a generar más del 70\% del PIB nacional; c) su gran metrópolis, Montevideo, está situada en la costa; d) la mayoría de sus puertos son de interés nacional; e) en esta metrópolis y esos puertos se concentra gran parte de la producción industrial y el comercio de productos de mayor valor, y f) sus áreas costeras constituyen el principal destino turístico de Uruguay y de países de su entorno, fundamentalmente Argentina y Brasil (PNUMA, CLAES y DINAMA, 2008; Viana, 2009), albergando casi al $80 \%$ de los turistas que llegan al país.

Paralelamente a este proceso, la zona costera de Uruguay ha ido sufriendo cambios de manera acelerada que se traducen en una serie de problemas y conflictos. La variedad de usos y actividades que se desarrollan en estos espacios la convierten en un área compleja y dinámica. Dichos problemas se asocian a formas y modalidades de uso y ocupación de la costa, que han encontrado su sustento en múltiples relaciones sociales, ambientales, económicas e institucionales (MCI-Sur, 2006: 9; Gómez, 2009: 268). Lo anterior induce a pensar que la presión sobre los servicios de los ecosistemas, siendo elevada en la actualidad, tiende a ser mayor en el futuro, razón por la cual el litoral de Uruguay requiere de iniciativas que contribuyan al uso más racional de dichos servicios.

Es por ello que desde los años 90 en el país se han desarrollado con muy distinto alcance, escala y éxito, una serie de experiencias tendentes a promover la gestión integrada de sus áreas litorales. Entre otras, además de Ecoplata, objeto de este trabajo, destacan los programas Freplata y Probides (Conde, 2006: 68).

El programa binacional (Uruguay-Argentina) Freplata se inicia en 2000 con el fin de encontrar fórmulas conjuntas para mejorar la gestión de los recursos costeros del Río de la Plata. El proyecto fue financiado por el Fondo de Naciones Unidas para el Ambiente Global (GEF). Los objetivos fueron prevenir y mitigar la degradación de los recursos transfronterizos del Río de la Plata y su Frente Marítimo; contribuir a su uso sostenible por los habitantes de ambos países ribereños. Además, establecieron realizar un análisis de diagnóstico transfronterizo que llenase una serie de vacíos de información y suministrase datos e instrumentos clave para la definición del programa de acción estratégica. Dicho plan incluía propuestas para las políticas, el marco legal e institucional, e inversiones prioritarias para el Río de la Plata y su frente marítimo, así como estrategias y planes específicos, objetivos y mecanismos de implantación para la prevención, reducción y control de la contaminación, protección y conservación de la biodiversidad.

Probides, por su parte, es un Programa interinstitucional integrado por el Ministerio de Vivienda, Ordenamiento Territorial y Medio Ambiente (MVOTMA); varias intendencias municipales y la Universidad de la República; además cuenta con el apoyo del Programa de las Naciones Unidas para el Desarrollo (PNUD). Su finalidad es la conservación de la bio- 
diversidad y el desarrollo sustentable en la región Este del Uruguay, y tiene como objetivos: contribuir a la conservación y uso sostenible de la biodiversidad; incentivar el desarrollo económico y social; colaborar en el ordenamiento territorial, y apoyar el desarrollo institucional y de las capacidades locales presentes en la región. Desde que inició sus actividades en 1993, se han ido ejecutando varios proyectos con recursos del Fondo para el Medio Ambiente Mundial (GEF), la Unión Europea, la Agencia Española de Cooperación Iberoamericana (AECI), el MVOTMA y contribuciones privadas.

\section{EL PROGRAMA ECOPLATA}

Actualmente, Ecoplata es un programa que tiene por objeto fortalecer en los aspectos vinculados con la gestión integrada de áreas litorales a las administraciones públicas, a la comunidad científica, a los gestores y a la sociedad uruguaya en general. El programa se basa en un acuerdo interinstitucional entre el Ministerio de Vivienda, Ordenamiento Territorial y Medio Ambiente, el Ministerio de Ganadería, Agricultura y Pesca, el Ministerio de Defensa Nacional y la Universidad de la República. Participan también la Dirección Nacional de Saneamiento y Aguas, la Dirección de Recursos Renovables, la Prefectura Nacional Naval y las Intendencias Municipales costeras (Colonia, San José, Montevideo, Canelones, Maldonado y Rocha).

DATOS GENERALES DEL PROGRAMA ECOPLATA (2006- 2009)

\begin{tabular}{|c|c|c|c|c|}
\hline 1. Nombre & \multicolumn{4}{|c|}{$\begin{array}{l}\text { Conectando el conocimiento con la acción para la gestión integrada de la zona costera } \\
\text { del Río de la Plata (Ecoplata) }\end{array}$} \\
\hline \multicolumn{2}{|c|}{ 2. Ubicación geográfica } & \multicolumn{3}{|c|}{ Zona costera de Uruguay } \\
\hline \multirow{2}{*}{\multicolumn{3}{|c|}{ 3. Superficie sujeta al esfuerzo de manejo $\left(\mathrm{km}^{2}\right)$}} & Área terrestre & 5.891 \\
\hline & & & Mar territorial & 140.297 \\
\hline \multicolumn{4}{|c|}{ 4. $\mathrm{km}$ de costa } & 714 \\
\hline \multicolumn{4}{|c|}{ 5. Población estimada en el área del Programa } & 1.832 .015 \\
\hline \multirow{2}{*}{\multicolumn{4}{|c|}{$\begin{array}{l}\text { 6. Duración } \\
\text { 7. Monto total de financiación (US \$) }\end{array}$}} & 2006-2009 \\
\hline & & & & 780.060 \\
\hline \multicolumn{5}{|c|}{ 8. Fuentes de financiación } \\
\hline \multicolumn{4}{|c|}{ International Development Research Centre - Canadá (IDRC) } & $73 \%$ \\
\hline \multicolumn{4}{|c|}{ Ministerio de Vivienda Ordenación del Territorio y Medio Ambiente (MVOTMA) } & $27 \%$ \\
\hline
\end{tabular}

Fuente: elaboración propia a partir de PNUMA, CLAES y DINAMA, 2008; Programa Ecoplata, 2006 a y 2010.

\section{IV.1. Origen y evolución}

Desde su constitución en 1991, tras la firma de un convenio entre el Gobierno de Uruguay y la Universidad Dalhousie de Halifax (Nova Scotia, Canadá), el Programa Ecoplata ha pasado por cuatro etapas que vienen a consolidar un ciclo. Dichas etapas se sintetizan a continuación: 


\section{Primera etapa (1991-1993)}

En este periodo se pretendía establecer vínculos entre las comunidades científicas de Uruguay y Canadá. Con lo cual, el Programa se orientó al fortalecimiento de las capacidades de investigación en las ciencias del mar (Martínez y Fournier, 1999). Así se abría un camino para que las instituciones participantes aunaran esfuerzos en torno a objetivos de trabajo comunes.

\section{Segunda etapa (1994-1996)}

Producto del anterior periodo fue la elaboración del Proyecto Ecoplata II (1994-1996). En esta etapa, con financiación del IDRC y las instituciones uruguayas, se pensó ya en establecer una iniciativa a largo plazo. En estos años se apostó por fortalecer y desarrollar la capacidad de las comunidades científica y pesquera con objeto de prevenir la degradación de los espacios y recursos costeros.

Con la finalidad de dar a conocer a los representantes de los diferentes actores implicados en la gestión costera los resultados obtenidos hasta el momento, y ampliar la iniciativa con la integración de nuevas instituciones, en 1996 se celebró la Conferencia Internacional Ecoplata'96: Hacia el desarrollo sostenible de la zona costera uruguaya del Río de la Plata. Fruto de este encuentro fue la aprobación de la Declaración de Montevideo sobre desarrollo sostenible en la zona costera, que recogía los principales acuerdos logrados en la reunión.

\section{Tercera etapa (1997-2001)}

En 1997 se aprueba Ecoplata III (1997-2001) con la finalidad de contribuir a la gestión integrada de los recursos de la zona costera uruguaya del Río de la Plata. A través de un proceso interactivo e interdisciplinario el programa pretende contribuir a la formulación de políticas y estrategias de gestión integrada, siendo desarrolladas por las instituciones gubernamentales en el ámbito de sus competencias, con la participación de los actores sociales involucrados. En esta fase se incorporaron líneas de investigación relacionadas con las ciencias sociales y se abordó las partes terrestres de la costa del Río de la Plata. También se llevan a cabo proyectos de GIZC a escala local con objeto de acumular aprendizajes en este nuevo enfoque de gestión, comenzando a trabajar en 1999 en dos áreas piloto (Arroyo Carrasco-Arroyo Pando y Playa Pascual-Punta Espinillo) a través de la investigación y la realización de acciones demostrativas (Aguirre et al., 2001; Programa Ecoplata, 2001).

\section{Cuarta etapa (2002-2009)}

En 2002, con apoyo del IDRC-Canadá y el respaldo de las instituciones participantes, se firmó el documento que ampliaba el proyecto hasta 2005. En este período los trabajos estuvieron encaminados a la realización de distintas evaluaciones, estudios y diagnósticos de la zona costera de Uruguay (MCI-Sur, 2006). Más adelante, el lema «conectar el conocimiento con la acción» expresa bastante bien la intención del proyecto por avanzar más en su ejecución (Programa Ecoplata, 2006a, 2006b, 2007, 2009a y 2009b).

Durante el quinquenio 2010-2015 el Programa Ecoplata se encuentra en la fase de institucionalización, según el modelo (UNESCO/COI, 2001). La adopción formal del programa de gestión es el fin de un proceso de legitimación iniciado desde la primera etapa. Gracias a este 
proceso, la gestión se ha de considerar parte de un contrato social, frente a los responsables técnicos y políticos; contrato que, sin duda alguna, se debería insertar en la estrategia nacional de desarrollo sostenible de las zonas costeras de Uruguay.

\section{IV.2. Objetivos y funcionamiento del Programa Ecoplata (2006-2009)}

Teniendo como objetivo general fortalecer la GIAL mediante un modelo de gobernanza para el desarrollo sustentable del litoral del Río de la Plata, los objetivos específicos son los que se detallan a continuación (Programa Ecoplata, 2007, 2009a y 2009b):

1. Favorecer la participación y coordinación de las instituciones y actores vinculados al espacio costero, en apoyo al desarrollo de un modelo de gobernanza para la GIAL que incorpore el conocimiento generado.

2. Generar y sistematizar conocimiento mediante la recopilación de información y promoción de investigadores aplicada y participativa en apoyo a modelos de gestión costera.

3. Coordinar las actividades técnicas inherentes al proyecto.

El programa es ejecutado por el MVOTMA y supervisado por una Junta Directiva y una Mesa Ejecutiva. Cuenta además con la Unidad de Coordinación que es la encargada de articular y coordinar a los diferentes actores implicados y realizar trabajos técnicos junto con determinados Grupos Técnicos Temáticos y Consultores.

La Junta Directiva desempeña las funciones de aprobar las políticas que orientan su funcionamiento, así como las fuentes de financiación, normas de selección de personal, promover y concertar acuerdos con otros organismos y establecer nuevos objetivos. Presidido por el MVOTMA, está constituida además por otros ministerios que también tienen relación con el espacio y los recursos costeros (Ganadería, Agricultura y Pesca; Defensa Nacional; Transporte y Obras Públicas; Turismo y Deporte), la Universidad de la República (Facultades de Ciencias, Ciencias Sociales, Arquitectura e Ingeniería), organismos internacionales (IDRC, PNUD y UNESCO) y las seis intendencias municipales con zona costera (Canelones, Colonia, Maldonado, Montevideo, Rocha y San José).

Por su parte, la Mesa Ejecutiva es la encargada de orientar y supervisar la puesta en marcha y desarrollo de las actividades del Proyecto. Presidida también por el MVOTMA, la componen la Dirección Nacional de Ordenamiento Territorial y la de Medioambiente, el Ministerio de Ganadería, Agricultura y Pesca, la Dirección Nacional de Recursos Acuáticos, Ministerio de Defensa Nacional, el Servicio de Hidrografía, Oceanografía y Meteorología de la Armada, la Universidad de la República (Facultad de Ciencias y Facultad de Ciencias Sociales), el IDRC, el PNUD, la UNESCO, el Programa de Ciencias Ecológicas, y dos representantes de las Intendencias Municipales de Canelones y Montevideo.

\section{DISCUSIÓN}

La consulta de la bibliografía reseñada, las entrevistas y las visitas técnicas realizadas a determinadas zonas costeras y organismos institucionales de la República de Uruguay nos permiten expresar algunas opiniones al respecto. 
El fin último, como se ha expuesto en páginas precedentes, no es otro que contribuir de forma modesta a una de las tareas, o necesidades, más importantes que todo programa de gestión costera debe prever: la evaluación que permita una posterior retroalimentación del mismo programa en fases ulteriores. Y es que las características intrínsecas de estos instrumentos de gestión costera implican continúas remodelaciones y adaptaciones (Barragán, 2005a).

En primer lugar, este trabajo se considera desde una perspectiva político-técnica con objeto de definir el marco institucional de gestión de las áreas litorales. Así se analiza la firma de convenios o acuerdos entre agentes institucionales y sociales implicados en la gestión integrada de zonas costeras. También, se estudia la parte relacionada con las tareas de planificación, es decir, el equipo técnico, los integrantes, socios y el apoyo gubernamental. Y por último, la formulación lógica que corresponde a una planificación estratégica: declaración de intenciones o de principios, redacción de la visión y la misión, objetivos, tareas, recursos, etc.

\section{V.1. Valoración del Programa Ecoplata}

\section{V.1.1. Valoración del equipo técnico}

La formación ideal de un grupo de gestores o técnicos costeros debe ser multidisciplinar. No cabe duda de que la capacitación es fundamental para abordar con éxito un cambio en el modelo de gestión costera. Dependiendo de la capacitación se tendrán dos concepciones bien diferenciadas de la GIAL: una muy ligada a las ciencias físicas y naturales «aproximación ecológica» (con evidentes sesgos hacia la biología, ecología, oceanografía, geomorfología, química, etc.). Y otra más orientada a las ciencias sociales «aproximación antropológica» (más relacionada con la gestión y administración pública, resolución de conflictos, técnicas de negociación, técnicas para la toma de decisiones, comunicación, economía de recursos, etc.). Con el paso del tiempo una y otra han ido cediendo parte de sus contenidos hasta producir una interesante fusión. En el caso de Ecoplata, se evidencia un desequilibrio entre estas aproximaciones, dominando el perfil de las formaciones orientadas a las ciencias sociales.

Tabla 3

VALORACIÓN DEL EQUIPO TÉCNICO

\begin{tabular}{|l|c|c|c|}
\hline Valoración & F & M & D \\
\hline ¿Es multidisciplinar? & & $\mathrm{X}$ & \\
\hline ¿Permite tener una visión holística? & & $\mathrm{X}$ & \\
\hline ¿Es competente y comprometido? & $\mathrm{X}$ & & \\
\hline
\end{tabular}

F: favorable; M: medio; D: desfavorable.

\section{V.1.2. Valoración de los integrantes y socios}

Como se ha comentado en la introducción, el modelo de gobernanza como nueva forma de gobierno consiste en implicar a los tres actores fundamentales: la sociedad, el mercado y el gobierno en la toma de decisiones. Por tanto, entre los integrantes y socios del Programa 
Ecoplata ${ }^{1}$ se echa en falta a los dos primeros. Respecto a la participación pública se constata la ausencia de mecanismos para involucrar a los agentes sociales en el proceso de toma de decisiones del programa. Es decir, en los dos órganos colegiados que supervisan, orientan y deciden sobre el desarrollo de Ecoplata, la Junta Directiva y la Mesa Ejecutiva, la representación es, casi en exclusiva, de tipo institucional. No existe representación de la sociedad civil (Pérez-Cayeiro, 2013). Por su parte, en relación con las escalas territoriales de la administración existentes en el país, estatal y local, se constata que ambas aparecen bien representadas. En este sentido se destaca que los seis municipios litorales están presentes en Ecoplata, a través de sus gobiernos locales, al igual que los ministerios más vinculados a la gestión de los recursos y el espacio litoral.

Tabla 4

VALORACIÓN DE LOS INTEGRANTES

\begin{tabular}{|l|c|c|c|}
\hline Valoración & F & M & D \\
\hline $\begin{array}{l}\text { ¿Están representados todos los actores institucionales y sociales con } \\
\text { competencias o intereses en la zona costera? }\end{array}$ & & $\mathrm{X}$ \\
\hline $\begin{array}{l}\text { ¿Están representadas todas las escalas territoriales de la administración? } \\
\text { (escalas nacional y local) }\end{array}$ & $\mathrm{X}$ & & \\
\hline
\end{tabular}

\section{V.1.3. Valoración de las sinergias creadas entre Ecoplata y otras iniciativas}

En la etapa evaluada han sido diversas y numerosas las colaboraciones entre Ecoplata y otras iniciativas relacionadas con el litoral del país. Destacan entre otras, Sistema Nacional de Áreas Protegidas, Maestría en Manejo Costero Integrado del Conosur, Red Nacional de Educación Ambiental para el Desarrollo Humano Sostenible, Proyecto «Implementing Pilot Climate Change Adaptation Measures in Coastal Areas of Uruguay», etc. En el ámbito internacional, además de Freplata, hay que destacar que Uruguay es miembro de la Red Iberoamericana de Manejo Costero Integrado (IBERMAR). Esta iniciativa, auspiciada por el Programa Iberoamericano de Ciencia y Tecnología para el Desarrollo (CYTED) y conformada por investigadores y gestores de 13 países, tiene dos objetivos específicos (Barragán, 2009 y 2011). Por un lado, establecer una plataforma de coordinación para el intercambio de conocimiento y experiencias orientadas al manejo costero integrado. Y por otro, formular y consensuar a escala regional un Programa Iberoamericano de Cooperación y Transferencia Científica y Tecnológica en Manejo Costero Integrado (Barragán, 2012).

1 Instituciones y socios integrados en el Programa Ecoplata: Universidad de la República del Uruguay; Dirección Nacional de Recursos Acuáticos; Servicio de Oceanografía, Hidrografía y Meteorología de la Armada; Prefectura Nacional Naval; Ministerio de Vivienda Ordenamiento Territorial y Medioambiente; Ministerio de Educación y Cultura; Ministerio de Ganadería Agricultura y Pesca; Ministerio de Turismo y Deporte y los gobiernos locales de las intendencias municipales de: Colonia, San José, Montevideo, Canelones, Maldonado y Rocha. Socios del Ecoplata: Programa de las Naciones Unidas para el Desarrollo Organización de las Naciones Unidas para la Educación, la Ciencia y la Cultura; Centro Internacional de Investigaciones para el Desarrollo Ministerio de Vivienda Ordenamiento Territorial y Medioambiente. 
Tabla 5

VALORACIÓN DE LAS SINERGIAS CREADAS EN ECOPLATA

\begin{tabular}{|l|c|c|c|}
\hline Valoración & F & M & D \\
\hline $\begin{array}{l}\text { ¿Ha habido esfuerzos de colaboración e intercambio de conocimiento } \\
\text { con otros proyectos/programas de ámbito nacional? }\end{array}$ & $\mathrm{X}$ & & \\
\hline $\begin{array}{l}\text { ¿Ha habido esfuerzos de colaboración e intercambio de conocimiento } \\
\text { con otros proyectos/programas de ámbito internacional? }\end{array}$ & & $\mathrm{X}$ & \\
\hline
\end{tabular}

\section{V.1.4. Valoración del planteamiento de planificación estratégica de la iniciativa}

A raíz de la revisión de la información técnica del programa, se pone de manifiesto que su planteamiento se corresponde con la lógica de una planificación estratégica. Así, la visión enuncia de forma detallada las mejoras institucionales, sociales y ambientales que se esperan. Estas mejoras están diseñadas con un horizonte a medio largo plazo para aprovechar e intercambiar aprendizajes, y para mejorar los usos y la calidad de vida de la población local de forma interdisciplinaria, interinstitucional y con una fuerte participación. Por su parte, la misión describe de qué manera el programa hace operativo su papel en el respaldo de la visión: apoyando a sus socios directos; identificando a los actores principales y especificando los instrumentos que se desarrollarán en la ejecución del mismo. También, los objetivos específicos se corresponden totalmente con el objetivo general del programa. Éstos cubren los asuntos que abarca la misión y son realistas teniendo en cuenta los recursos, la experiencia de las instituciones y el contexto político, económico y social en el que opera Ecoplata. Además, las tareas involucran a diferentes agentes sociales e institucionales y requieren esfuerzos de coordinación entre diversos grupos. Y por último, los productos esperados son resultados tangibles a corto y medio plazo.

Tabla 6

VALORACIÓN DEL PLANTEAMIENTO DE PLANIFICACIÓN ESTRATÉGICA

\begin{tabular}{|l|c|c|c|}
\hline Valoración & F & M & D \\
\hline ¿Existe una declaración explícita de la misión y visión? & $\mathrm{X}$ & & \\
\hline ¿Están definidas las metas y los objetivos del programa? & $\mathrm{X}$ & & \\
\hline ¿Existe un cronograma organizativo de tareas y resultados? & $\mathrm{X}$ & & \\
\hline
\end{tabular}

\section{V.1.5. Valoración de la implicación gubernamental}

Tanto en la Junta Directiva como en la Mesa Ejecutiva del programa Ecoplata hay una importante implicación gubernamental. De la revisión de las actas de seguimiento del programa se puede comprobar que la asistencia y participación de los miembros que constituyen ambos órganos es adecuada. Además, la periodicidad de la convocatoria es óptima. La Mesa Ejecutiva celebra una reunión al mes y la Junta Directiva realiza un encuentro cada seis meses. 
En cuanto a la representatividad y representación cabe destacar, por un lado, que la participación de los integrantes es representativa. Es decir, que los miembros institucionales que forman parte de ambos órganos tienen potestad para la toma de decisiones de los grupos a los que representan. Por otro, en la composición de las dos figuras faltan responsables de otros ministerios del gobierno vinculados con los recursos costeros. En este sentido, sería interesante incluir a otras instituciones de la administración pública que estén relacionadas directa o indirectamente con los recursos y el espacio litoral.

Tabla 7

VALORACIÓN DE LA IMPLICACIÓN GUBERNAMENTAL

\begin{tabular}{|l|c|c|c|}
\hline Valoración & F & M & D \\
\hline ¿Hay representatividad en la Junta Directiva de Ecoplata? & X & & \\
\hline ¿Hay representación en la Junta Directiva de Ecoplata? & & & $\mathrm{X}$ \\
\hline ¿Hay representatividad en la Mesa Ejecutiva de Ecoplata? & $\mathrm{X}$ & & \\
\hline ¿Hay representación en la Mesa Ejecutiva de Ecoplata? & & & $\mathrm{X}$ \\
\hline
\end{tabular}

\section{V.1.6. Valoración de la ejecución}

Del análisis y la revisión de los documentos técnicos, que se reseñan en la bibliografía y proporcionados por Ecoplata, se obtienen los siguientes resultados: el cronograma planificado ha sido cumplido; se han obtenido los resultados esperados y se ha hecho el seguimiento de las distintas actividades desarrolladas a través de indicadores y medios de verificación.

Tabla 8

VALORACIÓN DE LAEJECUCIÓN

\begin{tabular}{|l|c|c|c|}
\hline Valoración & F & M & D \\
\hline ¿La ejecución ha seguido la lógica del plan de gestión? & & $\mathrm{X}$ & \\
\hline $\begin{array}{l}\text { ¿El desarrollo de tareas ha sido suficientemente explícito para } \\
\text { permitir el análisis de su validez? }\end{array}$ & $\mathrm{X}$ & & \\
\hline ¿Se han alcanzado las metas y objetivos planteados? & & $\mathrm{X}$ & \\
\hline ¿Se han difundido los resultados obtenidos? & & $\mathrm{X}$ & \\
\hline ¿Se ha evaluado adecuadamente la ejecución de las tareas? & $\mathrm{X}$ & & \\
\hline ¿Se ha formulado un programa de seguimiento? & & $\mathrm{X}$ & \\
\hline
\end{tabular}

\section{V.1.7. Valoración de los beneficiarios ${ }^{2}$ de los resultados de Ecoplata}

Un gran número de actividades desarrolladas por el Programa Ecoplata, en esta etapa, están muy vinculadas a la educación ambiental y al uso turístico de las playas (regeneración de dunas, accesos, etc.). Por tanto, se echa en falta a grupos sociales ligados a otros inte-

2 Los beneficiarios de las iniciativas desarrolladas por Ecoplata son: Agentes sociales de las Intendencias: Colonia, Canelones, San José, Montevideo, Maldonado y Rocha; Universidades; ONGs (El Abrojo, CLAES, Organización Conservación de Cetáceos y Karumbé) y Centro Cultural de España en Uruguay. 
reses económicos (pescadores, agricultores, etc.). Es por ello, que sería de interés valorar en el diseño del programa de gestión litoral, una vez ejecutado, ¿qué grupos obtendrían los beneficios y qué grupos verían amenazados sus intereses? En algunos casos, aquellos que se benefician monetariamente y/o políticamente de los patrones prevalecientes de uso de los recursos estarían reacios a una iniciativa de gestión porque ello puede afectar a sus intereses.

Tabla 9

VALORACIÓN DE LOS BENEFICIARIOS DE LOS RESULTADOS DE ECOPLATA

\begin{tabular}{|l|c|c|c|}
\hline Valoración & F & M & D \\
\hline $\begin{array}{l}\text { ¿Se han beneficiado los agentes sociales e institucionales de forma } \\
\text { equilibrada en todo el territorio costero nacional? }\end{array}$ & & $\mathrm{X}$ & \\
\hline ¿Se han beneficiado agentes de la mayoría de los grupos de interés? & & & $\mathrm{X}$ \\
\hline
\end{tabular}

\section{V.1.8. Valoración de los cambios institucionales}

Finalizada la quinta etapa y puesta en marcha la institucionalización del programa se vislumbran cambios en las instituciones. Así, se encuentra en la Junta Directiva, creada por Ecoplata, un espacio de discusión y de toma de decisiones para la gestión integrada de áreas litorales. Por otro lado, el Plan Estratégico de Gestión Costera validado y sometido a aprobación, se ha elaborado siguiendo los principios de la Gobernanza de participación y consenso entre diferentes agentes sociales e institucionales. Es destacable la colaboración del Programa en la elaboración de las Directrices Nacionales del Espacio Costero, que serán la futura política nacional explícita para la zona costera. El Modelo de Unidad Gestora de las Intendencias tiene en cuenta el principio de subsidiariedad, colocando la toma de decisiones en la escala de gobierno más cercana al ciudadano. También, los numerosos Acuerdos Institucionales firmados garantizan el apoyo y el compromiso de los diferentes grupos de interés para un cambio de modelo en la gestión integrada de las zonas costeras. Y por último, las reuniones de los Grupos Técnicos Temáticos (GTT) facilitan la búsqueda de consenso en la toma de decisiones.

Tabla 10

VALORACIÓN DE LOS CAMBIOS INSTITUCIONALES

\begin{tabular}{|l|c|c|c|}
\hline Valoración & F & M & D \\
\hline $\begin{array}{l}\text { ¿Los resultados están provocando un cambio en la toma de } \\
\text { decisiones de forma más integrada? }\end{array}$ & $\mathrm{X}$ & \\
\hline $\begin{array}{l}\text { ¿Los resultados garantizan la creación de una figura institucional } \\
\text { que centralice la gestión de los asuntos costeros? }\end{array}$ & $\mathrm{X}$ & & \\
\hline $\begin{array}{l}\text { ¿Los resultados proveen recursos económicos y humanos que hagan } \\
\text { factible la implantación del plan estratégico y su posterior evaluación? }\end{array}$ & & $\mathrm{X}$ \\
\hline $\begin{array}{l}\text { ¿Los resultados han favorecido el establecimiento de procedimientos } \\
\text { de coordinación y participación en la escala nacional y en la escala } \\
\text { local? }\end{array}$ & $\mathrm{X}$ & \\
\hline
\end{tabular}




\section{V.1.9. Valoración de los cambios en los usos}

A partir de las iniciativas llevadas a cabo en esta etapa ${ }^{3}$, en términos generales, los cambios en los usos se pueden considerar positivos sobre todo desde una perspectiva de educación ambiental. Aunque, en algunos sectores productivos de la sociedad estos parecen ser más modestos.

Tabla 11

VALORACIÓN DE LOS CAMBIOS EN LOS USOS

\begin{tabular}{|l|c|c|c|}
\hline Valoración & F & M & D \\
\hline $\begin{array}{l}\text { ¿Los resultados han conseguido potenciar la concienciación y la } \\
\text { participación de los distintos sectores sociales? }\end{array}$ & X & \\
\hline ¿Los resultados están favoreciendo la mejora en el uso de los recursos? & & $\mathrm{X}$ & \\
\hline $\begin{array}{l}\text { ¿Los resultados están contribuyendo a la mejora de equipamientos } \\
\text { e infraestructuras? }\end{array}$ & $\mathrm{X}$ & & \\
\hline $\begin{array}{l}\text { ¿Los resultados están ayudando a aumentar el conocimiento de los } \\
\text { usuarios de los recursos y procesos naturales? }\end{array}$ & $\mathrm{X}$ & & \\
\hline
\end{tabular}

\section{V.1.10. Valoración de los cambios ambientales}

Las mejoras introducidas en el espacio litoral a través de los proyectos piloto en las diferentes intendencias han tenido como objetivo estabilizar dunas y crear accesos a la playa en Canelones, Maldonado y Rocha. No obstante, estas iniciativas parecen estar más relacionadas con una política turística que con una política ambiental. Es por ello que el Programa Ecoplata debería haber sido más ambicioso con la protección ambiental e introducir cambios desde el punto de vista urbanístico y de la ordenación del territorio en determinadas zonas.

Tabla 12

VALORACIÓN DE LOS CAMBIOS AMBIENTALES

\begin{tabular}{|l|c|c|c|}
\hline Valoración & F & M & D \\
\hline ¿Los resultados han mejorado unidades ambientales costeras? & & & $\mathrm{X}$ \\
\hline ¿Los resultados han mejorado el estado de los recursos costeros? & & & $\mathrm{X}$ \\
\hline
\end{tabular}

\section{V.2. Diagnóstico integrado de la GIAL en Uruguay}

A continuación se procede a la síntesis de los aspectos más relevantes de esta evaluación del Programa Ecoplata en el período 2006-2009. Con el análisis de cada uno de ellos, se ha pretendido desglosar las que se pueden denominar razones subyacentes. Por éstas se entienden aquellas causas últimas o profundas, que ayudan a detectar o a comprender mejor el funcionamiento de la iniciativa.

3 Gestión costera participativa en el Arroyo Pando, Modelo de gestión participativa en la localidad Colonia Wilson; I Foro de Desarrollo Sustentable de la Zona Costera; Encuentro de Jóvenes por la costa; Publicaciones; Informes técnicos para la toma de decisiones; Página web de Ecoplata (www.ecoplata.org); Cursos de capacitación a distintos sectores o el Sistema de Información Ambiental Costero (SIAC). 
Para ello se utiliza la matriz de análisis DAFO. Esta herramienta permite trabajar con información e ideas referidas al contexto: uno interno (Debilidades y Fortalezas) y otro externo (Amenazas y Oportunidades). Algunos autores como Medianero (2004) proponen su utilización como una herramienta de análisis estratégico que permite obtener una perspectiva general de la gestión institucional.

Tabla 13

ANÁLISIS DAFO DEL PROGRAMA ECOPLATA

\begin{tabular}{|c|c|}
\hline $\begin{array}{l}\text { Debilidades } \\
\text { - Ausencia de un soporte legal específico que res- } \\
\text { palde el cambio hacia un modelo más integrado } \\
\text { de gestión. } \\
\text { - Recursos insuficientes y dispersos para una ges- } \\
\text { tión más integrada de las zonas costeras. } \\
\text { - Ausencia de órganos colegiados para la partici- } \\
\text { pación y cooperación en asuntos litorales. } \\
\text { - Escasos recursos dedicados a programas espe- } \\
\text { cíficos de educación y concienciación. }\end{array}$ & $\begin{array}{l}\text { Amenazas } \\
\text { - Lentitud de los cambios institucionales que } \\
\text { contrasta con la rapidez de los procesos de de- } \\
\text { gradación del espacio y los recursos. } \\
\text { - Modelo reactivo de gestión pública. } \\
\text { - Origen externo de la financiación (organismos } \\
\text { internacionales). } \\
\text { - Carácter excesivamente formal y representativo } \\
\text { de la participación y cooperación. }\end{array}$ \\
\hline $\begin{array}{l}\text { Fortalezas } \\
\text { - Conciencia de las políticas públicas para asumir } \\
\text { un nuevo modelo de GIAL. } \\
\text { - Sentido incipiente de gobernanza que cala en la } \\
\text { acción de gobierno. } \\
\text { - Capacidad del Programa de institucionalizar y } \\
\text { fortalecer el proceso de GIAL. Ecoplata tiene } \\
\text { una trayectoria de más de } 15 \text { años. } \\
\text { - Vínculos establecidos entre el Programa y di- } \\
\text { versos agentes institucionales y sociales. } \\
\text { - Recopilación de información de gran interés y } \\
\text { en formatos que permiten la utilización de las } \\
\text { nuevas tecnologías. } \\
\text { - Experiencia en participación pública y coopera- } \\
\text { ción administrativa. } \\
\text { - Experiencia en programas educativos y de con- } \\
\text { cienciación sobre temas litorales. }\end{array}$ & $\begin{array}{l}\text { Oportunidades } \\
\text { - Futura aprobación de las Directrices Naciona- } \\
\text { les del Espacio Costero. } \\
\text { - Revitalización de la Comisión Oceanográfica } \\
\text { Uruguaya. } \\
\text { - Demanda social creciente para una gestión más } \\
\text { sostenible. } \\
\text { - Contexto nacional e internacional favorable } \\
\text { para iniciativas aplicadas a la GIAL. } \\
\text { - Elevada conciencia sobre la necesidad de coor- } \\
\text { dinación y cooperación institucional. } \\
\text { - Posibilidades de cooperación con las diferentes } \\
\text { escalas de la administración. } \\
\text { - Vínculos con otras iniciativas de GIAL nacio- } \\
\text { nales e internacionales. } \\
\text { - Apertura de canales específicos para la partici- } \\
\text { pación pública y la cooperación institucional. }\end{array}$ \\
\hline
\end{tabular}

\section{CONCLUSIONES}

En primer lugar hay que destacar que la cooperación internacional, en este caso de la mano de International Development Research Centre- Canada (IDRC) a través del patrocinio del Programa Ecoplata, ha conseguido generar una concienciación y un compromiso gubernamental inusuales en el litoral. Como expresión del entendimiento y la colaboración internacional entre ambas partes la gestión integrada de zonas costeras se interpreta como un área de gestión estratégica en Uruguay. Es decir, en este ejercicio de cooperación, un concierto de voluntades, se ha convertido en un instrumento potente para consolidar y hacer frente a la GIAL en el país con el transcurso de los años (Pérez-Cayeiro, 2013). 
Es también digno de resaltar los importantes esfuerzos realizados por el Programa Ecoplata para favorecer el entendimiento entre las dos escalas territoriales de la administración de la costa en Uruguay: estatal y municipal. A ello puede que haya contribuido en cierta manera la estabilidad social y económica que ha disfrutado el país en los últimos años y sus dimensiones terrestres y marítimas, no hay que olvidar que estamos ante un país de unos 700 km de costa y más de 3 millones de habitantes.

Otra reflexión se identifica con las conclusiones obtenidas después de nuestras visitas a las instituciones relacionadas con el Programa, las entrevistas realizadas y las actividades desarrolladas. En ellas se ha podido comprobar la aceptación que ha tenido el Programa en esta etapa.

A diferencia de otras iniciativas semejantes, el Programa Ecoplata ha conseguido escalar hasta la esfera gubernamental. Y por este motivo la iniciativa continúa, aún, cuando finaliza el apoyo y la financiación externa. No obstante, la voluntad política juega un papel fundamental para proseguir con los procedimientos ya establecidos, en este caso, desde hace más de una década. De todas formas, el reto de esta nueva etapa, sin duda, será comprobar que la GIAL es asumida con recursos propios. En la actualidad la iniciativa se alimenta de los presupuestos nacionales. En cierto modo, se puede considerar un gran paso en la institucionalización de la iniciativa. Y ello resulta fundamental para la consolidación de una política específica de las áreas litorales.

No se debe desaprovechar la oportunidad que Ecoplata representa para institucionalizar un modelo de desarrollo sostenible en las zonas costeras de Uruguay. Por ello cabe valorar positivamente dicha iniciativa en el sentido de que el MVOTMA ha sido capaz de dotarse de una moderna herramienta para la gestión de su zona costera. Ello está permitiendo consolidar una buena base para su gestión integrada.

La idea general que subyace en lo redactado anteriormente respecto al Programa Ecoplata es muy favorable. Esta iniciativa, como decisión y proyecto político orientado a la acción, «conectar el conocimiento con la acción», es de una enorme trascendencia para el litoral de Uruguay. Se debe resaltar que constituye una relevante aportación al diseño del aparato institucional que muy probablemente gestionará en el futuro las áreas litorales del país.

\section{BIBLIOGRAFÍA}

AGUILAR, L.F. (2006): Gobernanza y gestión pública. México. Fondo de Cultura Económica.500 pp.

AGUIRRE, M. et al. (2001): Una experiencia de gestión integrada costera. Programa Ecoplata 1999-2001. Montevideo. Programa Ecoplata. 125 pp.

BARRAGÁN, J.M. (2003): Medio ambiente y desarrollo en áreas litorales. Introducción a la Planificación y Gestión Integradas. Servicio de publicaciones Universidad de Cádiz. Cádiz. 301 pp.

BARRAGÁN, J.M. (2005 a): La gestión de las áreas litorales en Latinoamérica y España. Cádiz. Servicio de Publicaciones de la Universidad de Cádiz. 192 pp.

BARRAGÁN, J.M. (2005 b): «The coasts of Latin America at the end of the century». Journal of Coastal Research, $\mathrm{n}^{\circ} 17$ (4), 885-899. 
BARRAGÁN, J.M., CHICA, J.A. y PÉREZ-CAYEIRO, M.L. (2008): Propuesta de Estrategia Andaluza de Gestión Integrada de Zonas Costeras. Consejería de Medio Ambiente de la Junta de Andalucía. Cádiz. 255 pp.

BARRAGÁN, J.M. (coord.) (2009): Manejo Costero Integrado y Política Pública en Iberoamérica: Un diagnóstico. Necesidad de cambio. Red IBERMAR (CYTED), Cádiz. $380 \mathrm{pp}$.

BARRAGÁN, J.M. (coord.) (2011): Manejo Costero Integrado y Política Pública en Iberoamérica: Propuestas para la acción. Red IBERMAR (CYTED), Cádiz, 280 pp.

BARRAGÁN, J.M. (coord.) (2012): Manejo Costero Integrado y Política Pública en Iberoamérica: Diagnóstico y propuestas para una nueva política pública. Red IBERMA (CYTED), Cádiz, 152 pp.

CARVALLO, V.C. y RIZZO, H.G. (1994): A zona costeira brasileira. Subsídios para uma avaliaçâo ambiental. Ministerio de Medio Ambiente y de la Amazonia. Secretaría de coordinación de asuntos de Medio Ambiente. Brasilia. D.F.

CICIN- SAIN, B. y KNECH, R.W. (1998): Integrated coastal and ocean management. Concepts and practice. Island Press. Washington, D. C. 517 pp.

CLARK, J.R. (1992): Integrated management of coastal zones. FAO Fisheries Technical Paper, $\mathrm{n}^{\circ}$ 327. Roma. $167 \mathrm{pp}$.

COMISIÓN EUROPEA (2000): Comunicación de la Comisión al Consejo y al Parlamento Europeo sobre: La gestión integrada de las zonas costeras: una estrategia para Europa. Bruselas, COM (2000) 547 final. Bruselas.

COMISIÓN EUROPEA (1999): Hacia una estrategia europea para la gestión integrada de las zonas costeras. Principios generales y opciones políticas. Documento de reflexión. Luxemburgo. Oficina de Publicaciones Oficiales de las Comunidades Europeas. 35 pp.

CONDE, D. (2006): «Estudios de base sobre la situación del MCI en Uruguay» en MCISur. Conferencia Regional: Fortalecimiento de capacidades para el Manejo Costero Integrado. Proyecto «Sustentabilidad de la zona costera uruguaya» (AUCC-CICA). Montevideo, Universidad de la República, 67-74.

COUNCIL OF EUROPE (2000a): Model Law on Sustainable Management of Coastal Zones. Strasbourg, Council of Europe Publishing, Nature and Environmental Series, $\mathrm{n}^{\mathrm{o}}$ $101.27 \mathrm{pp}$.

COUNCIL OF EUROPE (2000b): European Code of Conduct for Coastal Zones. Strasbourg, Council of Europe Publishing, Nature and Environmental Series, $\mathrm{n}^{\circ} 101.84 \mathrm{pp}$.

GEE, K. et al. (2006): The role of spatial planning and ICZM in the sustainable development of coasts and seas. Final report. Integrated Coastal Zone Management (ICZM): Strategies for coastal and marine spatial planning. Berlin. Research project of the Federal Ministry of Transport, Buiding and Spatial Planning (BBR). 76 pp.

GESAMP (1999): La contribución de la ciencia al manejo costero integrado. Roma. FAO. $75 \mathrm{pp}$.

GÓMEZ, M. (2009): «Los asuntos clave para el manejo costero integrado en Iberoamérica: Uruguay» en Manejo costero integrado y política pública en Iberoamérica: Un diagnóstico. Necesidad de Cambio (Barragán Muñoz, J.M., coord.). Cádiz, Red Ibermar, CYTED, 261-290. 
GÓMEZ, M. et al. (2011): «Documento nacional de propuestas: Uruaguay» en Manejo costero integrado y política pública en Iberoamérica: Propuestas para la acción (Barragán Muñoz, J.M., coord.). Cádiz, Red Ibermar, CYTED, 191-217.

KAY, R. y ALDER, J. (1999): Coastal planning and management. London, E\&FN Spon. $370 \mathrm{pp}$.

MARTÍNEZ, C. M. y FOURNIER, R. (1999): «Ecoplata: an Uruguayan multi-institutional approach to integrated coastal zone management». Ocean Coastal Mangement, 42: 165-188.

MCI-SUR. (2006): Conferencia Regional: Fortalecimiento de capacidades para el Manejo Costero Integrado. Proyecto «Sustentabilidad de la zona costera uruguaya» (AUCCCICA), Universidad de la República, Montevideo. Dalhouse Uneversity, Halifax. 119 pp.

MEDIANERO, D. (2004): Guía metodológica para el diseño de planes estratégicos en el sector público. Facultad de Ciencias Económicas de la UNMSM. Perú. 32 pp.

MERINO CUESTA, M. (2010): Fundamentos de evaluación de políticas públicas, Agencia Estatal de Evaluación de las Políticas Públicas y la Calidad de los Servicios, Ministerio de Política Territorial y Administración Pública de España, 118 pp.

OCDE (1995): Gestión de Zonas Costeras. Políticas Integradas. Ediciones Mundi Prensa. Madrid. 204 pp.

OLMEDA, J. A. (1999): Ciencia de la administración (Volumen I). Teoría de la organización y gestión pública. Madrid, Universidad Nacional de Educación a Distancia.

OLSEN, S.B., LOWRY, K. y TOBEY, J. (1999): Una guía para evaluar el progreso en el manejo costero. Centro de Recursos Costeros de la Universidad de Rhode Island (CRCURI), Comisión Centroamericana de Ambiente y Desarrollo (CCAD) a través de su Proyecto Programa Ambiental Regional Centroamericano (PROARCA/Costas), Centro Regional para el Manejo de Ecosistemas Costeros (Ecocostas). Guayaquil. 71 pp.

OLSEN, S.B. (2003): «Frameworks and indicators for assessing progress in integrated coastal management initiatives». Ocean and Coastal Management, 46 (3-4): 347-361.

OLSEN, S.B. y OCHOA, E. (2004): Marco metodológico y para la planeación e implementación del manejo de ecosistemas costeros. Centro de Recursos Costeros de la Universidad de Rhode Island (CRC-URI) y Programa Ecocostas. Guayaquil. 23 pp.

PÉREZ-CAYEIRO, M.L. (2013): Gestión Integrada de Áreas Litorales: Evolución de la disciplina en las últimas dos décadas. Editorial Tebar, Madrid.

PROGRAMA ECOPLATA (2001): Conferencia Internacional ECOPLATA 2001 «estión Integrada de la Zona Costera Uruguaya del Río de la Plata». Programa ECOPLATA, Montevideo. 163 pp.

PROGRAMA ECOPLATA (2006a): Conectando el conocimiento con la acción para la gestión integrada de las zonas costeras. Documento de Proyecto URU/06/016. Documento mecanografiado. $18 \mathrm{pp}$.

PROGRAMA ECOPLATA (2006b): Plan Estratégico Ecoplata. Ecoplata, Montevideo. 58 pp.

PROGRAMA ECOPLATA (2007): Ecoplata rinde cuentas 2007. Unidad de Gestión Programa Ecoplata, Montevideo. 16 pp.

PROGRAMA ECOPLATA (2009a): Ecoplata rinde cuentas 2008. Unidad de Gestión Programa Ecoplata, Montevideo. 24 pp. 
PROGRAMA ECOPLATA (2009b): Ecoplata rinde cuentas 2009. Unidad de Gestión Programa Ecoplata, Montevideo. 24 pp.

PROGRAMA ECOPLATA (2010): Sostenibilidad de la gestión integrada en la zona costera del Uruguay. Conectando el Conocimiento con la acción. Montevideo. 72 pp.

PNUMA, CLAES y DINAMA (2008): Geo Uruguay 2008. Montevideo. PNUMA. 350 pp.

SUBIRATS, J., KNOEPFEL, P., LARRUE, C. y VARONE, F. (2012): Análisis y gestión de políticas públicas, Ed. Ariel, Barcelona, 285 pp.

UNEP (1995): Guidelines for Integrated Management of Coastal and Marine Areas. UNEP Regional Seas Reports and Studies, $\mathrm{n}^{\circ} 161.80 \mathrm{pp}$.

UNESCO/COI (2001): Steps and Tools towards integrated coastal area management. IOC Manuals and Guides, $\mathrm{n}^{\circ}$ 42. Paris. 64 pp.

UNESCO/COI (2003): A Reference Guide on the Use of Indicators for Integrated Coastal Management. ICAM Dossier 1, IOC Manuals and Guides n ${ }^{\circ} 45$. Paris. 127 pp.

UNESCO/COI (2006): A Handbook for Measuring the Progress and Outcomes of Integrated Coastal and Ocean Management. IOC Manuals and Guides, 46; ICAM Dossier 2. Paris. 217 pp.

VIANA MATTURRO, F. (2009): La zona costera de Uruguay: biodiversidad y gestión. Montevideo. Santillana. 159 pp. 